\title{
RESEARCH
}

Open Access

\section{Cardiac dysfunction in Multisystem Inflammatory Syndrome in Children: An Italian single-center study}

Savina Mannarino ${ }^{1 *}$, Irene Raso ${ }^{1}$, Massimo Garbin ${ }^{1}$, Elena Ghidoni ${ }^{1}$, Carla Corti ${ }^{1}$, Sara Goletto ${ }^{1}$, Luisa Nespoli ${ }^{1}$, Sara Santacesaria', Elena Zoia², Anna Camporesi², Francesca Izzo², Dario Dilillo ${ }^{3}$, Laura Fiori ${ }^{3}$, Enza D'Auria ${ }^{3}$, Annalisa De Silvestri ${ }^{4}$, Alberto Dolci ${ }^{5,6}$, Valeria Calcaterra ${ }^{3,7}$ and Gianvincenzo Zuccotti ${ }^{3,5}$

\section{Abstract}

Background: Multisystem inflammatory syndrome in children (MIS-C) is a novel condition temporally associated with SARS-CoV2 infection. Cardiovascular involvement is mainly evident as acute myocardial dysfunction in MIS-C. The aim of this study was to describe the cardiac dysfunction in patients with MIS-C, defining the role of severity in the clinical presentations and outcomes in a single cohort of pediatric patients.

Methods: A single-center retrospective study on patients diagnosed with MIS-C, according to the Center for Disease Control and Prevention (CDC) definition, and referred to Vittore Buzzi Children's Hospital in Milan from November 2020 to February 2021. Patients were managed according to a local approved protocol. According to the admission cardiac left ventricular ejection fraction (LVEF), the patients were divided into group A (LVEF < 45\%) and group B (LVEF $\geq 45 \%)$. Pre-existing, clinical, and laboratory factors were assessed for evaluating outcomes at discharge.

Results: Thirty-two patients were considered. Cardiac manifestations of MIS-C were reported in 26 patients (81\%). Group A included 10 patients (9 M/1F, aged 13 years [IQR 5-15]), and group B included 22 patients (15 M/7 M, aged 9 years [IQR 7-13]). Significant differences were noted among clinical presentations (shock, diarrhea, intensive care unit admission), laboratory markers (leucocytes, neutrophils, and protein C-reactive), and cardiac markers (troponin T and Nterminal pro B-type Natriuretic Peptide) between the groups, with higher compromission in Group A. We found electrocardiogram anomalies in 14 patients (44\%) and rhythm alterations in 3 patients (9\%), without differences between groups. Mitral regurgitation and coronary involvement were more prevalent in group A. Total length of hospital stay and cardiac recovery time were not statistically different between groups. A recovery of cardiac functioning was reached in all patients.

Conclusion: Despite significant differences in clinical presentations and need for intensive care, all of the MIS-C patients with significant cardiac involvement in this study completely recovered. This suggests that the heart is an involved organ and did not influence prognosis if properly treated and supported in the acute phase.

Keywords: Multisystem inflammatory syndrome, Children, Heart, Cardiac, COVID-19

\footnotetext{
* Correspondence: savina.mannarino57@gmail.com

'Pediatric Cardiology Unit, "V. Buzzi" Children's Hospital, 20154 Milan, Italy

Full list of author information is available at the end of the article
}

(c) The Author(s). 2021 Open Access This article is licensed under a Creative Commons Attribution 4.0 International License, which permits use, sharing, adaptation, distribution and reproduction in any medium or format, as long as you give appropriate credit to the original author(s) and the source, provide a link to the Creative Commons licence, and indicate if changes were made. The images or other third party material in this article are included in the article's Creative Commons licence, unless indicated otherwise in a credit line to the material. If material is not included in the article's Creative Commons licence and your intended use is not permitted by statutory regulation or exceeds the permitted use, you will need to obtain permission directly from the copyright holder. To view a copy of this licence, visit http://creativecommons.org/licenses/by/4.0/ The Creative Commons Public Domain Dedication waiver (http://creativecommons.org/publicdomain/zero/1.0/) applies to the data made available in this article, unless otherwise stated in a credit line to the data. 


\section{Background}

Multisystem inflammatory syndrome in children (MIS-C), also called pediatric inflammatory multisystem syndrome, is temporally related to SARS-CoV2 infection. It is a rare but severe hyperinflammatory disease that affects pediatric patients, typically 3-6 weeks after SARS-CoV2 contact [1].

While children are largely spared from severe acute SARS-CoV2 infection, probably due to under-expression of ACE2 receptors [2], MIS-C is a condition with clinical features similar to incomplete Kawasaki disease, macrophage activating syndrome, toxic shock syndrome, and septic shock $[3,4]$. It affects multiple organs, the cardiac, gastrointestinal, muco-cutaneous, respiratory, neurological, and hematological systems $[5,6]$.

MIS-C was first described in April 2020 in Europe, but nowadays reports and papers exist from all over the world. The incidence is generally unknown, but a recent US paper reported an adjusted estimated incidence from 1 to 10 cases per 1.000 .000 people per month [7]. Male patients and Hispanic and African people have a higher prevalence $[8,9]$.

The majority of patients are previously healthy children, without relevant comorbidities, with the exception of asthma and obesity [5].

The pathophysiological mechanism of the disease is unclear. MIS-C cases have a 3-6-week lag from the peak of cases in adults. The proposed mechanism is a postinfectious phenomenon mediated by IgG antibodies. Apparently, acute infection of SARS-CoV-2 induces a proinflammatory reaction and, in a genetically susceptible child, a delayed hyperinflammatory reaction of vasculitis with augmented levels of lymphocyte T-helper 17 and T-helper 1 mediators, and a cytokine storm, including massive release of inflammatory mediators and exaggerated activation of the immune system [10]. Chang et al., in a recent study, supported the role of a skewed cytokine profile towards IL6/IL8 pathways in causing heart dysfunction [11].

The clinical manifestations include persistent fever and involvement of at least two organ systems. Gastrointestinal symptoms, including vomiting, diarrhea, and abdominal pain, are reported in the vast majority of cases, from 50 to $100 \%$. Rash, mucositis, conjunctivitis, and peripheral edema are frequently observed, in 36$81 \%$ of cases. Neurological symptoms are usually mild (headache, confusion, and irritability) and occur in 12$56 \%$ of cases, but rarely can be more severe, including meningitis and seizures. Respiratory symptoms are not a significant part of the MIS-C presentation, but shortness of breath, cough, hypoxia, and respiratory distress have been documented in up to $65 \%$ of cases $[9,12,13]$.

Cardiac system involvement is frequently described and echocardiography, electrocardiography, and even laboratory abnormalities included. Acute myocardial dysfunction is the most prevalent, with generally mild or moderate depressed ejection fraction (EF). Only one third of patients present with severely reduced $\mathrm{EF}(\mathrm{EF}<30 \%)$. Cardiogenic or vasodilatory shock are described in up to a half of MIS$\mathrm{C}$ diagnoses, and require inotropic support, while $28 \%$ need mechanical circulatory support [5].

Coronary arteries dilation and aneurysms are frequently described, but the prevalence varies significantly among reports, and it is not always easy to understand the rate of coronary enlargement and involvement. The most recent cardiological review reports an incidence of $8-24 \%$ in the larger studies. Most of the coronary involvement is mild (dilation or small aneurisms, $\mathrm{Z}$ score 2.5-5) and transient, but there have been a few reports of large aneurysms (Z score $>10)$ [14].

The aim of this study was to describe cardiac involvement in patients with MIS-C, defining the role of severe cardiac dysfunction in determining the clinical presentation and outcomes in a single cohort of pediatric patients.

\section{Patients and methods \\ Patients}

We carried out a retrospective study at the Pediatric Department of the Children's Hospital, Vittore Buzzi, Milano, from the 1st of November 2020 to the end of February 2021. We included all children and adolescents who met the criteria of MIS-C diagnosis according to the Center for Disease Control and Prevention (CDC) [15].

For all patients, demographics and clinical characteristics, comorbidities, clinical presentation (fever duration, rash, conjunctivitis, extremities changes, mucositis, abdominal signs (diarrhea, vomiting, both), shortness of breath, headache, convulsions, shock), information about hospitalization (hospital stay length, need for pediatric intensive care unit (PICU), ventilation support, outcomes), and laboratory data (white blood cells, neutrophils, lymphocytes, C-reactive protein, N-Terminal pro B-type Natriuretic Peptide (NTproBNP), troponin T (high sensibility), ferritin, D-dimer, platelet, hemoglobin, interleukin-6) were recorded.

A cardiologic evaluation was carried out, including echocardiographic examinations and an electrocardiogram.

Mortality, length of hospital stay and LV systolic function at the discharge are considered as outcomes.

The study was conducted according to the guidelines of the Declaration of Helsinki, and approved by the Institutional Review Board of the Vittore Buzzi Hospital (Protocol number n. 2021/ST/004). All participants or their responsible guardians were asked for and gave their written consent after being informed about the nature of the study. 


\section{Methods}

\section{Clinical parameters}

The patient's physical examination included evaluation of weight, height, BMI (calculated as body weight in kilograms divided by height in meters squared) and pubertal stage according to Marshall and Tanner, as previously reported [16].

\section{Cardiologic assessment}

For all patients, we considered echocardiographic ventricular measurements at admission before and after intravenous immunoglobulin (IVIG) therapy and during the hospitalization if necessary, considering cardiac electric abnormalities and arrhythmias including heart block, and at discharge.

Left ventricular (LV) function was qualitatively and quantitatively assessed by echocardiogram (Philips Affinity 70 or Vivid S5 GE Healthcare), and the left ventricular ejection fraction (LVEF) was calculated according to the Simpson's biplane method [17].

To decide the therapeutic dosage of steroids, left cardiac function was classified as follows: normal (LVEF > 55\%), mildly reduced (LVEF 45-54\%), moderately reduced (LVEF 35-44\%), or severely reduced (LVEF < $35 \%)$, according to our protocol. We also measured mitral regurgitation (qualitative and semi-quantitative evaluation), the degree of pericardial effusion (mild if $\leq 5$ $\mathrm{mm}$, moderate if $>5 \mathrm{~mm}$ ), and the coronary artery size [18] (Montreal Z-score [19]).

According to the degree of compromised myocardial function, the patients were divided into two groups:

- Group A, patients with moderate and severe depression of the LVEF (LVEF < 45\%);

- Group B, patient without signs of cardiac involvement or with only mild depression of the LVEF (LVEF $\geq 45 \%$ ).

\section{Protocol therapy for MIS-C}

In all patients, our standard protocol for treatment [18, 20], based on a review of the current literature [21] and mainly derived from Kawasaki disease experience and multidisciplinary expert consensus, was adopted. The rationale of the therapy was to control and stop the hyperimmune response of MIS-C.

The cardinal treatment consisted of IVIG at $2 \mathrm{~g} / \mathrm{kg}$. Corticosteroids (methylprednisolone) were added in case of hemodynamic impairment, need for oxygen, and heart failure. In the case of mildly reduced LVEF or minimal oxygen support we gave methylprednisolone $2 \mathrm{mg} / \mathrm{kg}$ for 5 days, followed by gradual tapering. If there was a significant oxygen requirement, mild organ injury, and/or moderately reduced LVEF, methylprednisolone $10 \mathrm{mg} / \mathrm{kg}$ for 1 day then $2 \mathrm{mg} / \mathrm{kg}$ for 5 days was given before tapering. If there was a need for respiratory support, inotropic support, moderate to severe organ damage, and/ or severe hearing dysfunction, we used a high methylprednisolone dose of up to $30 \mathrm{mg} / \mathrm{kg}$ for 3 days.

Supportive care (inotropes, fluid resuscitation, diuretics, oxygen and ventilation, antibiotics, anticoagulation, or anti-thrombotic prophylaxis) were considered in most patients, accordingly to clinical multidisciplinary team evaluation. The anti-thrombotic prophylaxis was started in all patients $>12$ years old, and was considered in patients $<12$ years old, if the $\mathrm{D}$-dimer was high $(>5$ times the upper normal value) or if there was at least one known risk factors for thromboembolism. The anticoagulation therapy was prescribed in case of thrombosis and in case of severe LV dysfunction. With the reduction of the D-dimer or at the normalization of the LV function, heparin was shifted to low-dose aspirin for 3-4 weeks.

\section{Statistical analysis}

Quantitative values are described as the mean and standard deviation (SD), or the median and interquartile range if not normally distributed (Shapiro-Wilk test). Qualitative variables were described as counts and percentages. Comparisons between groups were made with a chi-square test for qualitative variables, and with a ttest or Mann-Whitney test for quantitative data.

\section{Results}

\section{General population data}

We identified 32 consecutive patients. The median age at admission was 10 years, ranging from 5 to 15 years (interquartile range, IQR, 7-13). The features of the patients are reported in Table 1 . Fever was present in all patients, with a median duration of 6 days at diagnosis. Nineteen patients $(41 \%)$ presented with more than two organs involved. No major common preexisting comorbidities were recorded. Four patients (13\%) were overweight and two patients (6\%) were obese. None of the reported patients had congenital heart disease or preexisting cardiovascular disease. All patients had positive IgG serology for the virus.

As expected, laboratory testing (Table 2) showed elevated inflammatory markers (C-reactive protein, fibrinogen, ferritin, D-dimer), neutrophilia with lymphopenia, and increased cardiac biomarkers.

All patients (100\%) were treated with IVIG at the time of diagnosis, with a median delay of $5 \pm 2$ days from the onset of symptoms. Treatment with steroids (methylprednisolone) was added for 30 patients (93.8\%): 5 (15.6\%) received the highest dose, 10 (31.3\%) the intermediate dose, and most (46.9\%) the lower dose, according to our protocol. 
Table 1 Baseline characteristics of the population, according to the degree of compromised myocardial function

\begin{tabular}{|c|c|c|c|c|}
\hline Baseline characteristics & Total & $\begin{array}{l}\text { Group A } \\
\text { LVEF }<45 \%\end{array}$ & $\begin{array}{l}\text { Group B } \\
\text { LVEF } \geq 45 \%\end{array}$ & $p$-value \\
\hline Patient numbers $(\mathrm{n})$ & 32 & 10 & 22 & \\
\hline Age (years), median (IQR) & $10(7-13)$ & $13(5-15)$ & $9(7-13)$ & ns \\
\hline Gender & & & & ns \\
\hline Male, n (\%) & $24(75)$ & $9(90)$ & $15(68)$ & \\
\hline Female, n (\%) & $8(25)$ & $1(10)$ & $7(32)$ & \\
\hline Overweight, n (\%) & $4(12.4)$ & $2(20)$ & $2(9.5)$ & ns \\
\hline Ethnicity, n (\%) & & & & ns \\
\hline Caucasian & $24(75)$ & $6(60)$ & $18(82)$ & \\
\hline African & $2(6.3)$ & $1(10)$ & $1(5)$ & \\
\hline Asian & $1(3.1)$ & $0(0)$ & $1(5)$ & \\
\hline Hispanic/Latino & $5(15.6)$ & $3(30)$ & $2(9,5)$ & \\
\hline Organs ystems involved, n (\%) & & & & ns \\
\hline 2 & $13(40.6)$ & $4(40)$ & $7(32)$ & \\
\hline 3 & $10(31.3)$ & $3(30)$ & $8(36)$ & \\
\hline 4 & $8(25)$ & $3(30)$ & $5(23)$ & \\
\hline 5 & $1(3.1)$ & $0(0)$ & $2(9)$ & \\
\hline RT-PCR positive, n (\%) & $2(6.3)$ & $2(20)$ & $0(0)$ & 0.03 \\
\hline Serology-positive, n (\%) & $32(100)$ & $10(100)$ & $22(100)$ & - \\
\hline Fever, n (\%) & $32(100)$ & $10(100)$ & $22(100)$ & - \\
\hline Fever duration (days) & 6 & 5.3 & 6.9 & 0.02 \\
\hline Gastrointestinal n (\%) & $30(94)$ & $10(100)$ & $20(91)$ & ns \\
\hline Vomit & $19(59)$ & $5(50)$ & $14(64)$ & ns \\
\hline Diarrhea & $20(63)$ & $9(90)$ & $11(50)$ & 0.04 \\
\hline Abdominalpain & $19(59)$ & $6(60)$ & $13(59)$ & ns \\
\hline Dermatological (rash), n (\%) & $14(44)$ & $6(60)$ & $8(36)$ & ns \\
\hline Shock, n (\%) & $8(25)$ & $6(60)$ & $2(9)$ & $<0.01$ \\
\hline Conjiuntivitis, n (\%) & $18(56)$ & $5(50)$ & $13(59)$ & ns \\
\hline Respiratory, n (\%) & $13(41)$ & $5(50)$ & $7(32)$ & ns \\
\hline Extremitychanges, n (\%) & $7(22)$ & $3(30)$ & $4(18)$ & ns \\
\hline Changes in the lips and oral cavity, $\mathrm{n}(\%)$ & $8(25)$ & $3(30)$ & $5(23)$ & ns \\
\hline Neurological, n (\%) & $5(16)$ & $1(10)$ & $4(18)$ & \\
\hline Headache & $3(9)$ & $1(10)$ & $2(9)$ & ns \\
\hline Convulsion & $3(9)$ & $1(10)$ & $2(9)$ & ns \\
\hline Hospital stay (days) & 13 & 14.5 & 11.5 & ns \\
\hline Pediatric Intensive Care Unit admission, n (\%) & $22(69)$ & $10(100)$ & $12(54,5)$ & 0.01 \\
\hline Pediatric Intensive Care Unit stay (days) & 2 & 4.6 & 2 & 0.02 \\
\hline Non invasive ventilation, $\mathrm{n}(\%)$ & $12(37.5)$ & $5(50)$ & $7(31.8)$ & ns \\
\hline Ventilation (days) & 2.5 & 3.2 & 2.9 & ns \\
\hline Inotropic support, n (\%) & $11^{\mathrm{a}}(34.4)$ & $7(70)$ & $4(18.2)$ & $<0.001$ \\
\hline
\end{tabular}

LVEF Left ventricular ejection fraction; PICU Pediatric intensive care unit

${ }^{a}$ All of them were supported with epinephrine, and 2 received also norepinephrine and levosimendan 
Table 2 Biochemical characteristics of the enrolled patients, according to the degree of compromised myocardial function

\begin{tabular}{|c|c|c|c|c|}
\hline Laboratory variable & All & Group A $(n=10)$ & Group B $(n=22)$ & $\mathbf{p}$ \\
\hline White blood cells ( $\left.\mathrm{n}^{\circ} / \mathrm{mm} 3\right)$ & $9350(6620-13,740)$ & $13.740(11.073-17.555)$ & $7.130(6.260-9.390)$ & 0.001 \\
\hline Neutrophil (n\%mm3) & $8050(5240-11,770)$ & $12.150(9.470-14.630)$ & $5.600(4.875-8.235)$ & 0.001 \\
\hline Lymphocytes (n\%mm3) & $840(650-1110)$ & $1.005(720-1.620)$ & $750(595-995)$ & ns \\
\hline Platelets (n\%mm3) & $165,000(112750-219,500)$ & $185.500(163.000-230.000)$ & 144.500 (102.000-191.000) & ns \\
\hline Hemoglobin (g/dl) & $10.8(10.23-11.95)$ & $10.6(10-12)$ & $11(10-12)$ & ns \\
\hline Ferritin $(\mu \mathrm{g} / \mathrm{L})$ & 875 (389.25-1498.5) & $788(374-1.924)$ & $1.007(419-1.470)$ & ns \\
\hline C-reactive protein (mg/L) & 200.8 (119.48-279.3) & $283(261-308)$ & $178(92-241)$ & 0.008 \\
\hline Troponin T (ng/L) & $37(14-74)$ & $72(40-243)$ & $22(8-49)$ & 0.01 \\
\hline NT-proB-type Natriuretic Peptide (ng/L) & $10,194.5(1562-15,441.3)$ & $14.825(11.340-17.810)$ & $5.921(1.114-11.243)$ & 0.01 \\
\hline D-Dimer $(\mu \mathrm{g} / \mathrm{L})$ & $2777(2126-5418)$ & $2.855(2.195-4.996)$ & $2.756(2.146-5019)$ & ns \\
\hline linterleukin-6 (ng/L) & 159 (97-405) & $253(156-579)$ & $110(96-155)$ & ns \\
\hline
\end{tabular}

Low-molecular-weight heparin was prescribed for 29 patients (91\%), of whom 7 (22\%) received a therapeutic anticoagulation dosage.

All patients were discharged with low dose aspirin and $93.8 \%$ with oral corticosteroid.

The majority of patients (69\%) were admitted to the PICU. No patients required intubation and mechanical ventilation, while $37.5 \%$ needed non-invasive respiratory support.

\section{Cardiac involvement}

Cardiac manifestations of MIS-C were reported in 26 patients $(81 \%)$ and included ventricular dysfunction, valvular regurgitation, coronary artery dilation, arrhythmia, and conduction abnormalities.

The echocardiogram showed 9 (28\%) children with preserved EF, 13 (41\%) with mildly reduced function, and $5(16 \%)$ and $5(16 \%)$ with moderately and severely reduced function, respectively. Mitral regurgitation was present in 23 cases (72\%), of whom 1 (3\%) was severe and $4(13 \%)$ moderate.

Only one child (3\%) developed a transient coronary ectasia of the main left, with a Boston $\mathrm{Z}$ score $<2.5$.

We found electrocardiogram anomalies in 14 patients (44\%), without a difference between groups. ST segment changes and/or negative $\mathrm{T}$ waves were recorded in 12 (38\%); 3 patients (9\%) developed a type-1 Brugada pattern, one of which also presented with atrial fibrillation (AF) during placement of the central venous catheter, electrically cardioverted. One patient showed a type 2 $\mathrm{BrS}$ pattern. None of them had a positive family history neither for sudden cardiac death nor for Brugada syndrome.

Rhythm anomalies occurred in three patients (9\%), including first-degree heart block, escape junctional rhythm alternating with sinus rhythm with premature ventricular contractions, and a short coupling interval. One patient presented with atrial fibrillation.
Ventricular dysfunction normalized in all our children in a median of $3 \pm 2$ days after treatment. Arrhythmias and electric abnormalities also tended to resolve.

\section{Cardiac involvement according to severity of dysfunction}

In our population, 10 patients belonged to group $\mathrm{A}$ and 22 to group B. There were no significant differences in terms of baseline characteristics between the two groups, Table 1.

Group B had a less frequent presentation in shock at admission (group A $60 \%$ vs group B $9 \%, p<0.01$ ) and lower incidence of diarrhea (group A $90 \%$ vs group B $50 \%, p=0.04$ ). Group A presented with a shorter duration of fever ( 5.3 vs 6.9 days, $p=0.02$ ).

All patients from group A were admitted to the PICU due to shock ( $100 \%$ vs $55 \%, p=0.01)$ and/or significant cardiac dysfunction. Compared to group B patients admitted to the PICU, group A patients spent more time in the PICU (4.6 days vs 2 days, $p=0.02$ ), and with a higher need for inotropic support (70\% vs $18 \%, p<0.01$ ), mainly epinephrine. There were no differences between the groups in terms of mortality, ventilation support $(p=\mathrm{ns})$, and hospital stay $(p=\mathrm{ns})$.

In the pretreatment echocardiographic examination, group A showed a significantly increased prevalence of mitral regurgitation ( $90 \%$ vs $64 \%, p<0.01$ ); the prevalence of pericardial effusion was not different between the groups. In only one group A patient was a coronary dilation recorded; however, it was transient and did not reach the criteria for aneurysm. For further details, see Table 3.

As reported in Table 2, a statistically significant difference was detected in the white blood cell count $(p=$ $0.001)$, number of neutrophils $(p=0.001)$, value of $C$ reactive protein $(p<0.01)$, troponin $\mathrm{T}(p=0.001)$, and NTproBNP levels $(p=0.01)$, with higher values in group A compared to B. On the contrary, the two groups did not differ in lymphocytes $(p=n s)$, platelets $(p=n s)$, 
Table 3 Echocardiographic findings pre treatment according to the degree of compromised myocardial function

\begin{tabular}{llll}
\hline Echocardiographic findings & Group A & Group B & $\boldsymbol{p}$-value \\
\hline LVEF $255 \%, \mathrm{n}(\%)$ & $0(0)$ & $9(41)$ & $<0.001$ \\
LVEF 45-54\%, n (\%) & $0(0)$ & $13(59)$ & $<0.001$ \\
LVEF 36-44\%, n (\%) & $5(50)$ & $0(0)$ & $<0.001$ \\
LVEF $\leq 35 \%, \mathrm{n}(\%)$ & $5(50)$ & $0(0)$ & $<0.001$ \\
Coronary dilation, n (\%) & $1(10)$ & $0(0)$ & - \\
Mitral regurgitation, n (\%) & $9(90)$ & $14(64)$ & $<0.01$ \\
Pericardial effusion, n (\%) & $4(40)$ & $3(14)$ & ns \\
\hline
\end{tabular}

LVEF Left Ventricular Ejection Fraction

hemoglobin $(p=\mathrm{ns})$, ferritin $(p=\mathrm{ns})$, D-dimer $(p=\mathrm{ns})$, and IL-6 $(p=0.08)$ values.

Pathological levels of troponin and NTproBNP were detected in all patients of group A. Furthermore, altered troponin and NTproBNP values were also noted in 33 and $77 \%$ of group B subjects, respectively. In Fig. 1, the trend of cardiac biomarkers in groups A and B during hospitalization were showed.

\section{Cardiac involvement during hospitalization}

At the echocardiographic evaluation $24-48 \mathrm{~h}$ after the end the therapy with IVIG and during steroid therapy, all patients except one of group A (LVEF pre IVIG 33\% after $35 \%$ ) had an LVEF $>45 \%$, without a significant mean difference between the two groups (group A 52.3\% vs group B $55.4 \%, \mathrm{p}=\mathrm{ns}$ ). Mild or moderate mitral regurgitation persisted in $60 \%(6 / 10)$ of group A and $64 \%$ $(14 / 22)$ of group B, while mild pericardial effusion persisted in 2/10 (20\%) patients of group $A$ and in $4 / 22$ (18\%) of group B, $\mathrm{p}=\mathrm{ns}$.

At discharge, all patients had an LVEF > 55\%, 10/32 (3\%) a mild mitral regurgitation, and 6/32 (1.9\%) a negligible pericardial effusion.

\section{Discussion}

The recent pandemic of COVID-19 was characterized by poor clinical involvement of children compared to adults, but a novel MIS-C, mediated by cytokine activation, has been reported. This syndrome arises 3-5 weeks after no evidence of active current COVID-19 infection, and causes systemic multi-organ inflammation. In midMay 2020, the CDC published a case definition for MIS$\mathrm{C}$ for disease surveillance [15]. The heart is one of the main organs involved, and cardiovascular complications triggered recommendations for immunomodulatory treatments. Ventricular dysfunction, pericardial effusion, and valvulitis are commonly diagnosed in MIS-C.

We reported cardiac involvement in $81 \%$ of our patients, confirming that the heart, together with the gastrointestinal system, represents the most common target organ in patients with MIS-C. Only $28 \%$ of children showed a preserved EF, $41 \%$ patients had a mildly reduced $\mathrm{EF}$, and 16 and $16 \%$ exhibited moderately and severely depressed functioning, respectively. A recent review of cardiac involvement in patients with MIS$\mathrm{C}$ reported left ventricular dysfunction in 31 to $58 \%$ of patients [14]; similarly, in a recent study, Leora R. Feldstein et al. reported $34.2 \%$ of patients had a depressed LVEF [12].

The age distribution of our population supports a greater involvement of older children and adolescents, (median age of 10 years), as already reported in the literature $[9,22]$.

Fortunately, in accordance with other previous studies [23-25], we observed a prompt response to therapy ( $3 \pm$ 2 days after treatment). At discharge, all patients had an LVEF $>55 \%$.

It is known that most patients recover within days to weeks and mortality is rare, although the medium- and long-term sequelae, and particularly cardiovascular complications, are not yet known [14, 22, 26].

A systematic review and meta-analysis reported a rate of $28.1 \%$ of different types of ECG abnormalities as atrial or ventricular arrhythmias [27]. William Regan et al. showed ECG changes in a majority of children (67\%) [28]. We found ECG abnormalities in $45 \%$ of patients, mainly abnormalities of repolarization, and one of the three cases with a type-1 Brugada pattern presented AF on the first day of hospitalization in intensive care. An Ajmaline test 8 months later confirmed Brugada Syndrome (not yet published). All patients at discharge presented a normal ECG, confirming a fast resolution of electrical anomalies and suggesting reversible damage.

In our pediatric cohort, only one Hispanic patient developed a transient ectasia of the main left coronary, which quickly resolved after therapy. There are conflicting descriptions about the data regarding the involvement of coronary arteries; the incidence of coronary artery dilatation/aneurysms varies significantly among reports $(8-24 \%)[14,29]$, and the pathological mechanism is, as yet, unknown. Abrams et al. showed some degree of association between muco-cutaneous alterations, conjunctival hyperemia, and coronary artery alterations, suggesting a possible overlap between MIS-C and Kawasaki disease [22]. None of our 23 patients with mucocutaneous manifestations had coronary involvement; many factors could have contributed to this difference, such as a strict selection of patients (all showed serological positivity of $\operatorname{IgG}$ ), a short interval between the onset of symptoms and therapy (median delay of $5 \pm 2$ days), combined therapy with steroids and intravenous immunoglobulin in almost patients $(>90 \%)$, and the prevalence of Caucasian ethnicity. Finally, the accurate recognition of patients with MIS-C may have limited overlap with Kawasaki disease. 


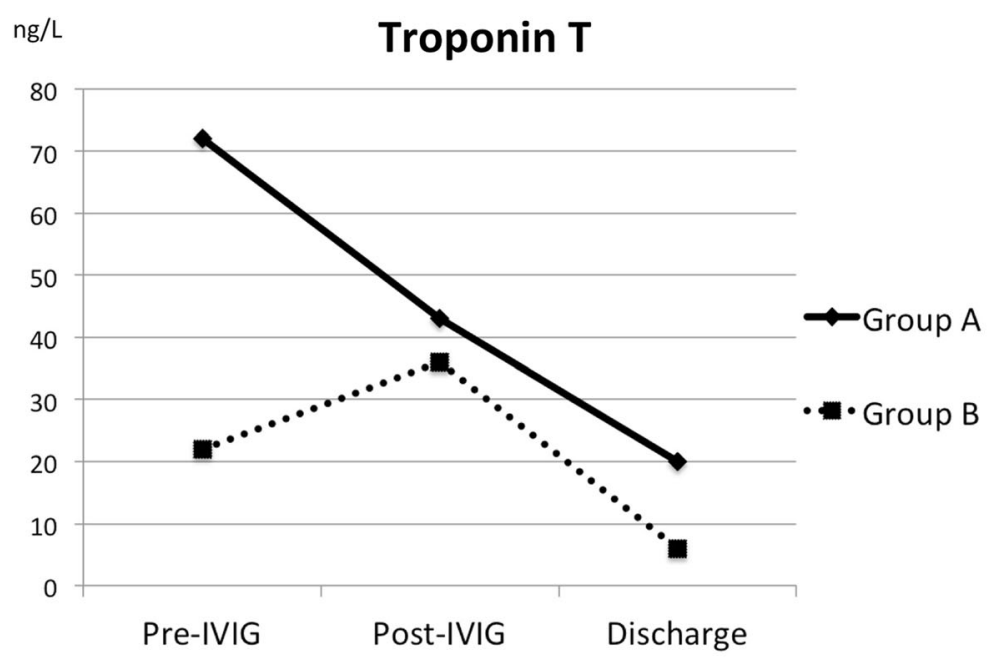

$\mathrm{ng} / \mathrm{L} \quad \mathrm{N}$-terminal pro b-type natriuretic peptide

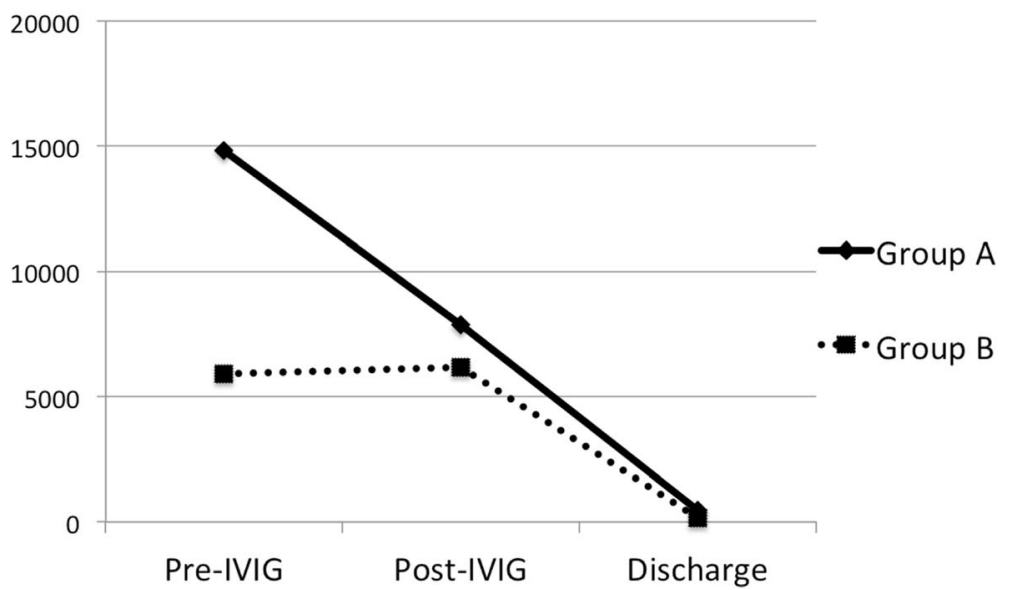

Fig. 1 Trend of cardiac biomarkers in groups A (LVEF < 45\%) and B (LVEF > 45\%); during hospitalization. LVEF = left ventricular ejection fraction; IVIG = intravenous immunoglobulin

We divided the patients in two groups according to severity of cardiac dysfunction on admission, to assess if there was any relationship between clinical and laboratory factors. All our patients had an alteration in the inflammatory index, with CRP, white blood cell, and neutrophil counts significantly higher in patients with more severe cardiovascular involvement; this suggests that myocardial involvement could be the hallmark of a hyperinflammatory state [5].

As expected, cardiac laboratory indices, including troponin $\mathrm{T}$ and NTproBNP levels, were higher in group A. Furthermore, values out of the ranges were also found in group B, suggesting that an underestimation of cardiac injury was not excluded using only echocardiographic evaluation.
Interestingly, the median value of NTproBNP was very high in group B as well. It is known that NTproBNP is associated with myocardial damage, stress, and fibrosis, and high NTproBNP levels constitute an independent diagnostic criterion for heart failure. However, it is also known that inflammation appears to be associated with natriuretic peptide release [30,31]. Increasing NTproBNP in MIS- $C$ without severe cardiac involvement may be related to the significant inflammatory state. Two patients in our series with shock at presentation showed a normal EF $(52-60 \%)$ and troponin $(49-46 \mathrm{ng} / \mathrm{L})$, and had very high NTproBNP values (17.810-23.748 ng/L) and PCR (456$500 \mathrm{mg} / \mathrm{L}$ ). As reported, shock and hemodynamic compromise in MIS-C can occur with preserved cardiac function. 
We noted that patients with more compromised heart function spent more time in resuscitation, however there were no differences between the groups in terms of mortality, ventilation support, and hospital stay. Additionally, LVEF recovery in group A patients did not differ from that in group B, indicating that the cardiac impairment at admission did not affect the therapeutic response. Even though, according to our protocol, more severe patients were treated with high-dose corticosteroids, the limited number of patients did not allow us to estimate the real effect of the different dosages.

\section{Limitations of the study}

We recognize that the limited sample size limits the strength of the analysis. Moreover, our results are similar to the current literature. Additionally, we were unable to perform global longitudinal strain analyses at the admission and check cardiac involvement with MRI during hospitalization, therefore an underestimation of cardiac injury should be considered. Finally, evaluation of long term heart consequences may be useful to define the real impact on children's cardiac health and cardiac MRI are likely indicated in the patients' follow-up.

\section{Conclusion}

Despite significant differences in clinical presentation and the need for intensive care, MIS-C patients with significant cardiac involvement completely recovered from illness without differences in term of cardiac function and length of hospital stay. There is a strict relationship between inflammatory state and cardiac impairment. We confirmed that the heart is one of the main target organs; however, when properly treated and supported in the acute phase, the cardiac involvement does not appear to affect the prognosis.

\section{Abbreviations}

AF: Atrial fibrillation; CDC: Center for disease control and prevention; IQR: Interquartile range; IVIG: Intravenous immunoglobulin; LVEF: Left ventricular ejection fraction; MIS-C: Multisystem inflammatory syndrome in children; NTproBNP: N-terminal pro b-type natriuretic peptide; PICU: Pediatric intensive care unit; SD: Standard deviation

\section{Acknowledgements}

Not applicable.

\section{Authors' contributions}

Conceptualization: S.M., I.R., M.G.; methodology, S.M., I.R., M.G., E.G., C.C., S.G., L.N., S.S., E.Z., A.C., F.I., D.D., L.F., E.D., A.D.S, A.D., V.C., G.Z; formal analysis, A.D.S.; investigation, S.M., I.R., M.G., E.G., C.C., S.G., L.N., S.S., E.Z., A.C., F.I, D.D., L.F., E.D., A.D.; writing—original draft preparation, S.M., I.R., E.G., A.D.S., V.C.; writing-review and editing, S.M., A.D.S., V.C., G.Z., C.M.; supervision, S.M., G.Z. All authors have read and agreed to the published version of the manuscript.

\section{Funding}

None.

\section{Availability of data and materials}

The datasets used and/or analysed during the current study are available from the corresponding author on reasonable request.

\section{Declarations}

\section{Ethics approval and consent to participate}

The study was conducted according to the guidelines of the Declaration of Helsinki, and approved by the Institutional Review Board of the Vittore Buzzi Children's Hospital (Protocol number n. 2021/ST/004). All participants or their responsible guardians were asked for and gave their written consent after being informed about the nature of the study.

\section{Consent for publication}

All participants or their responsible guardians gave their written consent for pubblication.

\section{Competing interests}

The authors declare that they have no competing interests.

\section{Author details}

'Pediatric Cardiology Unit, "V. Buzzi" Children's Hospital, 20154 Milan, Italy. ${ }^{2}$ Anesthesia and Intensive Care Unit, "Vittore Buzzi" Children's Hospital, 20154 Milan, Italy. 3Pediatric Department, "V. Buzzi" Children's Hospital, 20154 Milan, Italy. ${ }^{4}$ Biometry \& Clinical Epidemiology, Scientific Direction, Fondazione IRCCS Policlinico San Matteo, 27100 Pavia, Italy. ${ }^{5}$ Department of Biomedical and Clinical Science "L. Sacco", University of Milan, 20157 Milan, Italy. ${ }^{6}$ Pediatric and Adolescent Unit, Clinical Pathology Laboratory, "Luigi Sacco" Hospital, 27100 Pavia, Italy. Pediatric and Adolescent Unit,

Department of Internal Medicine, University of Pavia, 27100 Pavia, Italy.

Received: 13 October 2021 Accepted: 30 November 2021

Published online: 08 February 2022

References

1. Feldstein LR, Rose EB, Horwitz SM, Collins JP, Newhams MM, Son MBF, et al. Multisystem inflammatory syndrome in U.S. children and adolescents. N Engl J Med. 2020;383(4):334-46. https://doi.org/10.1056/NEJMoa2021680.

2. Calcaterra G, Mehta JL, Fanos V, Bassareo PP. Insights on Kawasaki disease and multisystem inflammatory syndrome: Relationship with COVID-19 infection. Minerva Pediatr. 2021:203-8 Available from: http://www.minerva medica.it. Accessed 3 Nov 2021.

3. Verdoni L, Mazza A, Gervasoni A, Martelli L, Ruggeri M, Ciuffreda M, et al. An outbreak of severe Kawasaki-like disease at the Italian epicentre of the SARS-CoV-2 epidemic: an observational cohort study. Lancet. 2020;395: 1771-8. Available from. https://doi.org/10.1016/S0140-6736(20)31103-X.

4. Riphagen S, Gomez X, Gonzalez-Martinez C, Wilkinson N, Theocharis P. Hyperinflammatory shock in children during COVID-19 pandemic. Lancet. 2020;395(10237):1607-8. Available from:. https://doi.org/10.1016/S0140-673 6(20)31094-1.

5. Belhadjer Z, Méot M, Bajolle F, Khraiche D, Legendre A, Abakka S, et al. Acute heart failure in multisystem inflammatory syndrome in children in the context of global SARS-CoV-2 pandemic. Circulation. 2020;142(5):429-36. Available from: http://ahajournals.org. https://doi.org/10.1161/CIRCULA TIONAHA. 120.048360.

6. Nakra NA, Blumberg DA, Herrera-Guerra A. Lakshminrusimha S. Multi-system inflammatory syndrome in children (Mis-c) following sars-cov-2 infection: Review of clinical presentation, hypothetical pathogenesis, and proposed management. Children. 2020; Available from: www.mdpi.com/journal/ children. Accessed 3 Nov 2021.

7. Payne AB, Gilani Z, Godfred-Cato S, Belay ED, Feldstein LR, Patel MM, et al. Incidence of Multisystem Inflammatory Syndrome in Children Among US Persons Infected With SARS-CoV-2. JAMA Netw Open. 2021;4:e2116420 Available from: https://jamanetwork.com/. Accessed 3 Nov 2021.

8. Stierman B, Abrams JY, Godfred-Cato SE, Oster ME, Meng L, Yip L, et al. Racial and Ethnic Disparities in Multisystem Inflammatory Syndrome in Children in the United States, March 2020 to February 2021. Pediatr Infect Dis J. 2021;Publish Ah:1-7.

9. Abrams JY, Godfred-Cato SE, Oster ME, Chow EJ, Koumans EH, Bryant B, et al. Multisystem inflammatory syndrome in children associated with severe acute respiratory syndrome coronavirus 2: a systematic review. J 
Pediatr. 2020;226:45-54.e1. Available from. https://doi.org/10.1016/j.jpeds.202 0.08.003.

10. Chen MR, Kuo HC, Lee YJ, Chi H, Li SC, Lee HC, et al. Phenotype, Susceptibility, Autoimmunity, and Immunotherapy Between Kawasaki Disease and Coronavirus Disease-19 Associated Multisystem Inflammatory Syndrome in Children. Front Immunol. 2021:632890 Available from: www. frontiersin.org. Accessed 3 Nov 2021.

11. Chang JC, Matsubara D, Morgan RW, Diorio C, Nadaraj S, Teachey DT, et al. Skewed Cytokine Responses Rather Than the Magnitude of the Cytokine Storm May Drive Cardiac Dysfunction in Multisystem Inflammatory Syndrome in Children. J Am Hear Assoc. 2021;10:21428 Available from: https://www.ahajo. Accessed 3 Nov 2021.

12. Feldstein $L R$, Tenforde MW, Friedman KG, Newhams M, Rose EB, Dapul $H$, et al. Characteristics and Outcomes of US Children and Adolescents With Multisystem Inflammatory Syndrome in Children (MIS-C) Compared With Severe Acute COVID-19. JAMA. 2021;325:1074-87 Available from: https://ja manetwork.com/. Accessed 3 Nov 2021.

13. Rafferty MS, Burrows H, Joseph JP, Leveille J, Nihtianova S, Amirian ES. Multisystem inflammatory syndrome in children (MIS-C) and the coronavirus pandemic: Current knowledge and implications for public health. J Infect Public Health. 2021:484-94 Available from: https:/reader.elsevier.com/reader/sd/pi/S1876034121000125?token= B15E49C12C3BE457D2D86F21CB772FE9DC7694660CBC15ADB18A1036C4D1FA7B1 649BB8887BD14EB2CBCC99DOC51BA44\&originRegion=eu-west-1\&originCreation= 20211006145021. Accessed 3 Nov 2021.

14. Alsaied T, Tremoulet AH, Burns JC, Saidi A, Dionne A, Lang SM, et al. Review of cardiac involvement in multisystem inflammatory syndrome in children. Circulation. 2021;143(1):78-88. https://doi.org/10.1161/CIRCULATIONAHA.12 0.049836 .

15. Thacker D. Multisystem Inflammatory Syndrome in Children (MIS-C). Delaware J Public Heal. 2020;6:36-9.

16. Calcaterra V, Klersy C, Vinci F, Regalbuto C, Dobbiani G, Montalbano C, et al. Rapid progressive central precocious puberty: diagnostic and predictive value of basal sex hormone levels and pelvic ultrasound. J Pediatr Endocrinol Metab. 2020:33(6):785-91. https://doi.org/10.1515/jpem-2019-0577.

17. Lopez L, Colan SD, Frommelt PC, Ensing GJ, Kendall K, Younoszai AK, et al. Recommendations for Quantification Methods During the Performance of a Pediatric Echocardiogram: A Report From the Pediatric Measurements Writing Group of the American Society of Echocardiography Pediatric and Congenital Heart Disease Council. J Am Soc Echocardiogr. 2010:465-95 Available from: https://reader.elsevier.com/reader/sd/pii/S0894731710002 66X?token=703D827A64991DCFC60DF6706BF9BB4181C9BEDDF26D92A CFEA4E1 1FE81ECA3FEDAA1E2C1231 AEEFE816E6E366A0BE19\&originRegion= eu-west-1\&originCreation=20211006121251. Accessed 3 Nov 2021.

18. Fuse S, Kobayashi T, Arakaki Y, Ogawa S, Katoh H, Sakamoto N, et al. Standard method for ultrasound imaging of coronary artery in children. Pediatr Int. 2010; 52(6):876-82. https://doi.org/10.1111/j.1442-200X.2010.03252.x.

19. Dallaire F, Dahdah N. New equations and a critical appraisal of coronary artery Z scores in healthy children. J Am Soc Echocardiogr. 2011;24(1):60-74. https://doi.org/10.1016/..echo.2010.10.004.

20. Cattalini M, Taddio A, Bracaglia C, Cimaz R, Della PS, Filocamo G, et al. Childhood multisystem inflammatory syndrome associated with COVID-19 (MIS-C): a diagnostic and treatment guidance from the Rheumatology Study Group of the Italian Society of Pediatrics. Ital J Pediatr. 2021;47. Available from(1):24. https://doi.org/10.1186/s13052-021-00980-2.

21. Jonat B, Gorelik M, Boneparth A, Geneslaw AS, Zachariah P, Shah A, et al. Multisystem inflammatory syndrome in children associated with coronavirus disease 2019 in a Children's Hospital in new York City: patient characteristics and an institutional protocol for evaluation, management, and follow-up. Pediatr Crit Care Med. 2021;22(3):E178-91. https://doi.org/10.1097/PCC 0000000000002598

22. Abrams JY, Oster ME, Godfred-cato SE, Bryant B, Datta SD, Campbell AP, et al. Factors linked to severe outcomes in multisystem inflammatory syndrome in children ( MIS-C) in the USA : a retrospective surveillance study. Lancet child Adolesc Heal; 2021;4642:10-12. Available from: http://dx. doi.org/https://doi.org/10.1016/S2352-4642(21)00050-X, 5

23. Ouldali N, Toubiana J, Antona D, Javouhey E, Madhi F, Lorrot M, et al. Association of intravenous immunoglobulins plus methylprednisolone vs immunoglobulins alone with course of fever in multisystem inflammatory syndrome in children. JAMA - J Am Med Assoc. 2021;325(9):855-64. https:// doi.org/10.1001/jama.2021.0694.
24. Hejazi Ol, Loke Y-H, Harahsheh AS. Short-term Cardiovascular Complications of Multi-system Inflammatory Syndrome in Children (MIS-C) in Adolescents and Children. Curr Pediatr Rep. 2021;1:3. Available from. https://doi.org/10.1 007/s40124-021-00258-5.

25. Wu EY, Campbell MJ. Cardiac manifestations of multisystem inflammatory syndrome in children (MIS-C) following COVID-19. Curr Cardiol Rep. 2021:3. Available from. https://doi.org/10.1007/s11886-021-01602-3.

26. Valverde I, Singh Y, Sanchez-de-Toledo J, Theocharis P, Chikermane A, Di Filippo S, et al. Acute cardiovascular manifestations in 286 children with multisystem inflammatory syndrome associated with COVID-19 infection in Europe. Circulation. 2021;143(1):21-32. https://doi.org/10.1161/CIRCULA TIONAHA. 120.050065.

27. Haghighi Aski B, Manafi Anari A, Abolhasan Choobdar F, Zareh Mahmoudabadi R, Sakhaei M. Cardiac abnormalities due to multisystem inflammatory syndrome temporally associated with Covid-19 among children: a systematic review and meta-analysis. IJC Hear Vasc. 2021:100764. Available from. https://doi.org/10.1016/j.jjcha.2021.100764.

28. Regan W, O'Byrne L, Stewart K, Miller O, Pushparajah K, Theocharis P, et al. Electrocardiographic changes in children with multisystem inflammation associated with COVID-19: associated with coronavirus disease 2019. J Pediatr. 2021;234:27-32.e2. Available from. https://doi.org/10.1016/j.jpeds.202 0.12 .033$.

29. Vella LA, Rowley AH. Current Insights Into the Pathophysiology of Multisystem Inflammatory Syndrome in Children. Curr Pediatr Rep. 2021;1:3. Available from. https://doi.org/10.1007/s40124-021-00257-6.

30. Fish-Trotter H, Ferguson JF, Patel N, Arora P, Allen NB, Bachmann KN, et al. Inflammation and Circulating Natriuretic Peptide Levels. Circ Hear Fail. 2020; 13:135-44 Available from: https://www.ahajournals.org/doi/suppl/10.1161/ CIRCHEARTFAILURE.119.006570. Accessed 3 Nov 2021.

31. Ganguly M, Nandi A, Banerjee P, Gupta P, Sarkar SD, Basu S, et al. A comparative study of IL-6, CRP and NT-proBNP levels in post-COVID multisystem inflammatory syndrome in children (MISC) and Kawasaki disease patients. Int J Rheum Dis. 2021; Available from: https://onlinelibrary. wiley.com/doi/10.1111/1756-185X.14236. Accessed 3 Nov 2021.

\section{Publisher's Note}

Springer Nature remains neutral with regard to jurisdictional claims in published maps and institutional affiliations.

Ready to submit your research? Choose BMC and benefit from

- fast, convenient online submission

- thorough peer review by experienced researchers in your field

- rapid publication on acceptance

- support for research data, including large and complex data types

- gold Open Access which fosters wider collaboration and increased citations

- maximum visibility for your research: over $100 \mathrm{M}$ website views per year

At BMC, research is always in progress.

Learn more biomedcentral.com/submissions 